\title{
Pure spin current manipulation in antiferromagnetically exchange coupled
}

heterostructures

L. Avilés-Félix, A. Butera, D. E. González-Chávez, R. L. Sommer, and J. E. Gómez

Citation: Journal of Applied Physics 123, 123904 (2018); doi: 10.1063/1.5023459

View online: https://doi.org/10.1063/1.5023459

View Table of Contents: http://aip.scitation.org/toc/jap/123/12

Published by the American Institute of Physics

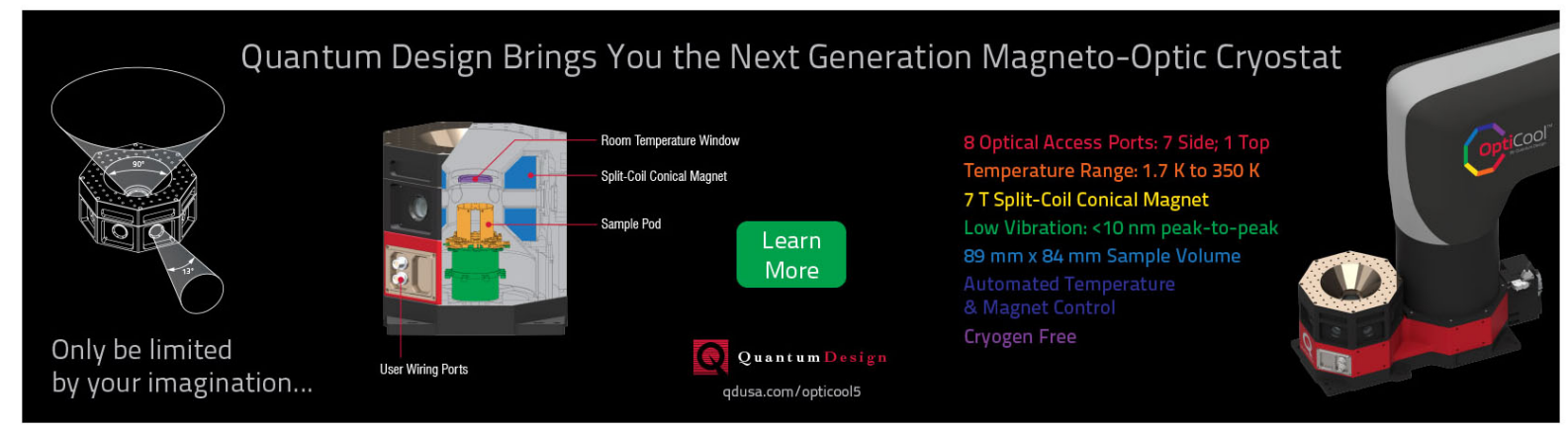




\title{
Pure spin current manipulation in antiferromagnetically exchange coupled heterostructures
}

\author{
L. Avilés-Félix, ${ }^{1, a), b)}$ A. Butera, ${ }^{1, a)}$ D. E. González-Chávez, ${ }^{2}$ R. L. Sommer, ${ }^{2}$ \\ and J. E. Gómez ${ }^{1}$ \\ ${ }^{1}$ Consejo Nacional de Investigaciones Científicas y Técnicas (Conicet), CABA, Argentina and Instituto \\ de Nanociencia y Nanotecnología, Centro Atómico Bariloche CNEA, Bariloche, Rio Negro, Argentina \\ ${ }^{2}$ Centro Brasileiro de Pesquisas Físicas, Rio de Janeiro, Brazil
}

(Received 24 January 2018; accepted 9 March 2018; published online 28 March 2018)

\begin{abstract}
We present a model to describe the spin currents generated by ferromagnet/spacer/ferromagnet exchange coupled trilayer systems and heavy metal layers with strong spin-orbit coupling. By exploiting the magnitude of the exchange coupling (oscillatory RKKY-like coupling) and the spinflop transition in the magnetization process, it has been possible to produce spin currents polarized in arbitrary directions. The spin-flop transition of the trilayer system originates pure spin currents whose polarization vector depends on the exchange field and the magnetization equilibrium angles. We also discuss a protocol to control the polarization sign of the pure spin current injected into the metallic layer by changing the initial conditions of magnetization of the ferromagnetic layers previously to the spin pumping and inverse spin Hall effect experiments. The small differences in the ferromagnetic layers lead to a change in the magnetization vector rotation that permits the control of the sign of the induced voltage components due to the inverse spin Hall effect. Our results can lead to important advances in hybrid spintronic devices with new functionalities, particularly, the ability to control microscopic parameters such as the polarization direction and the sign of the pure spin current through the variation of macroscopic parameters, such as the external magnetic field or the thickness of the spacer in antiferromagnetic exchange coupled systems. Published by AIP Publishing. https://doi.org/10.1063/1.5023459
\end{abstract}

\section{INTRODUCTION}

Conventional antiferromagnets (AF) and synthetic antiferromagnets (SAF) have been extensively used in engineered spintronic devices over the years, mainly for the storing, writing, and reading of information. Recent advances in the use of $\mathrm{AF}$ and SAF for the generation and detection of spin currents are playing a crucial role in the design of new spintronic devices. ${ }^{1,2}$ In addition to being easily adaptable to spintronics, some advantages of the use of crystal antiferromagnets involve the lack of stray fields as a consequence of its zero net magnetic moment in the absence of an external magnetic field; in the case of SAF, it is possible to control the exchange coupling between ferromagnetic thin films through metallic layers, to artificially obtain "pinned" electrodes in tunnel junction-like devices.

In a more recent context, it has been demonstrated that the use of antiferromagnets in the generation and detection of spin currents provides new ways to inject, propagate and detect pure spin currents, in some cases with improved efficiency. Recent works, both theoretical ${ }^{3-5}$ and experimental, ${ }^{6-10}$ highlight the role of $\mathrm{AF}$ not only as spin current injectors but also as angular momentum propagators when they are combined with ferromagnetic/heavy metal layered (FM/ML) structures. On the one hand, spin transport through

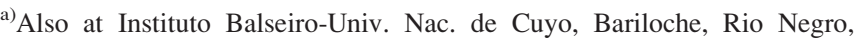
Argentina

b) Author to whom correspondence should be addressed: lavilesf@gmail.com
}

AF layers such as $\mathrm{NiO},{ }^{6} \mathrm{FeMn},{ }^{8}$ or $\mathrm{IrMn}^{11}$ inserted into $\mathrm{FM} /$ ML bilayers evidenced that it is possible to propagate spin currents through AF efficiently and also to enhance the spin to charge current conversion in the metallic layer. Khymyn et al. ${ }^{12}$ attributed the enhancement of the inverse spin Hall effect (ISHE) signal to the presence of a pair of evanescent AFM spin wave modes that can either attenuate or enhance the spin current depending on the phase difference between the evanescent spin wave modes. On the other hand, recent works explored theoretically a more active role of crystal antiferromagnets in spintronic devices. ${ }^{3,4}$ The use of AF as spin current injectors into adjacent normal metals with strong spin-orbit coupling (SOC) could lead to an enhancement of the spin Hall signal near the spin-flop transition in uniaxial antiferromagnets. The papers of Cheng et $\mathrm{al}^{3}{ }^{3}$ and Johansen and Brataas ${ }^{4}$ estimate the magnitude of the spin Hall voltages through the calculation of the spin current pumped from compensated $\mathrm{AF} / \mathrm{ML}$ interfaces in the $\mathrm{THz}$ region. This description of spin pumping (SP) also explores the influence of the ratio of the anisotropic energy to the exchange energy on the spin dynamics of AF/ML systems for the determination of a suitable antiferromagnet candidate for spin current generation.

The generation and detection of pure spin currents via spin pumping (SP) and the inverse spin Hall effect (ISHE) became an important research field in spin dependent transport. These effects can be used to estimate microscopic parameters related to the transport properties of $\mathrm{FM} / \mathrm{ML}$ 
systems. For example, the spin diffusion length $\left(\lambda_{S D}\right)$, the spin Hall angle $\left(\Theta_{S H}\right)$ and the spin mixing conductance $\left(g^{\uparrow \downarrow}\right)$ provide a way to determine the spin-to-charge current conversion efficiency in different $5 d$ transition metal elements such as $\mathrm{Pt}^{13,14}$ or $\mathrm{Ta},{ }^{15,16}$ which determines the suitable candidate for technological applications. The strong SOC in these transition metals is responsible for the ISHE, which converts a pure spin current into a transversal charge current according to $\mathbf{J}_{\mathrm{c}}=\Theta_{S H}\left(\frac{-2 e}{\hbar}\right) \mathbf{J}_{\mathbf{s}} \times \boldsymbol{\sigma}$, where $\mathbf{J}_{\mathrm{c}}$ and $\mathbf{J}_{\mathbf{s}}$ are the charge current and the pumped spin current, respectively, and $\sigma$ is the polarization direction of the pure spin current. Recent works in this field combine non-conventional components for the exploitation of these effects such as manganites $^{17,18}$ or magnetoelectric structures combined with strong SOC metals that can be used to modify the magnetic field position at which the spin pumping phenomenon occurs. ${ }^{19}$ The interplay between materials with different properties and metallic layers with strong SOC can lead to the design of new hybrid spintronic devices with improved performance. For example, the work of Johansen and Brataas ${ }^{4}$ also states that the strength of the spin pumping signal is influenced by the exchange interaction in $\mathrm{AF} / \mathrm{ML}$ systems.

In this framework, it is known that exchange interactions can also be used to control the interlayer exchange coupling (IEC) between two ferromagnetic layers through nonmagnetic metallic spacers (S). This exchange coupling is similar to the RKKY interaction, whose sign and magnitude depend on the spacer thickness, and it is mediated by the conduction electrons of the metallic spacer. This type of interaction is present in trilayer systems such as $\mathrm{Co} / \mathrm{Ru} /$ $\mathrm{Co},{ }^{20-22} \mathrm{Fe} / \mathrm{Au} / \mathrm{Fe}^{23}$ or $\mathrm{Py} / \mathrm{Cu} / \mathrm{Py} .{ }^{24}$

In this work, we will make use of the IEC between FM layers in order to modify the magnetization orientation during the resonance condition that can be used to control the polarization of the pure spin currents generated during the spin pumping process of the SAF/ML system. Our previous work ${ }^{25}$ reported the observation of spin pumping and the inverse spin Hall effect in antiferromagnetically coupled $[\mathrm{Co} / \mathrm{Ru} / \mathrm{Co}] / \mathrm{Pt}$ heterostructures. The spin-flop magnetization process observed in antiferromagnetically exchange coupled layers allowed us to control the polarization of the pure spin current injected into a heavy metal layer with strong SOC. In this paper, we aim to explain the behavior of a synthetic antiferromagnet as a spin current injector into a heavy metal layer through a phenomenological model that considers the influence of the spin-flop magnetization process on the spin pumping into the metallic layer. The model not only provides further insight into the spin-tocharge current conversion in SAF/ML systems, but also predicts the expected results during SP-ISHE experiments.

\section{EXPERIMENTAL CONSIDERATIONS}

Conventional spin current detection generated by spin pumping during the magnetic resonance condition of FM/ ML systems is carried out by measuring the voltage induced by the ISHE acting on the spin current that is "pumped" from the FM into a metallic layer with strong SOC. The ISHE signals in bilayer systems are commonly acquired by measuring the transversal voltage between the edges of the sample as described in previous works. ${ }^{13,26}$ In our case, the presence of antiferromagnetically exchange coupled structures, in which the magnetization is not necessarily aligned with the external magnetic field across the resonance condition, opens up the possibility to detect charge currents in unconventional directions. A schematic of the SAF-ML system and the electrical contact configuration for the detection of the pure spin currents via ISHE is shown in Fig. 1. The experiments are commonly performed inside of a resonance cavity with the external magnetic field $\mathbf{H}$ and the microwave magnetic field $\mathbf{h}_{\mu \omega}$ directions perpendicular to each other.

\section{MODEL}

In this section, we will discuss the magnetization dynamics, the spin pumping and the inverse spin Hall effect in SAF/ ML heterostructures. For the determination of the resonance modes and the equilibrium angles of the magnetization of the exchange coupled FM/S/FM system, we used a model adapted from Zhang et al. ${ }^{21}$ On the other hand, we propose a phenomenological model that describes the spin-to-charge current conversion of the pure spin current injected from a SAF into a ML, taking into consideration the influence of the spin-flop magnetization process on the spin pumping into the metallic layer as a mechanism for the polarization of the pure spin current. Our model also discusses the mechanism and the features for the detection of the induced ISHE voltages in SAF/ML in non-conventional directions via in-plane angular measurements of ISHE signals.

\section{A. Resonance modes and equilibrium angles in FM/S/ FM systems}

The magnetization dynamics of synthetic antiferromagnets can be described by using the free energy expression of

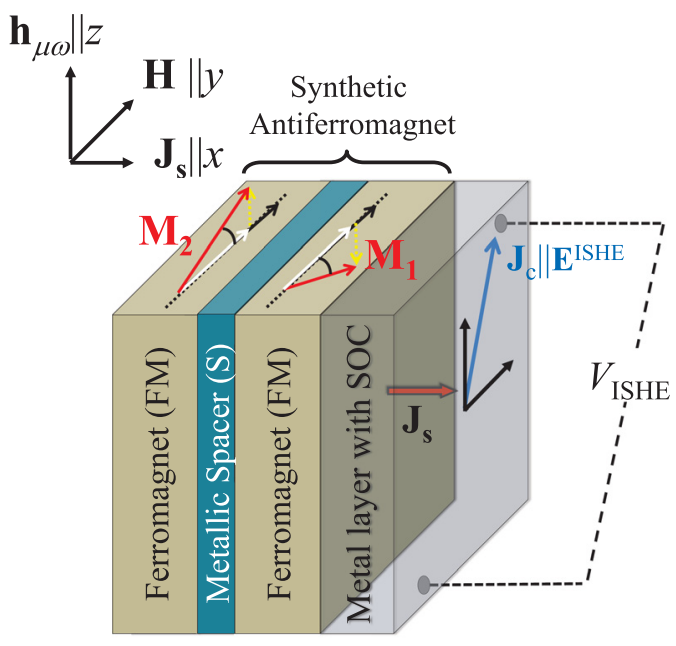

FIG. 1. Schematic of the detection mechanism of a pure spin current in a SAF/ML heterostructure via the inverse spin Hall effect. In the resonance condition, magnetization pumps a spin current $\mathbf{J}_{\mathbf{s}}$ into the metallic layer along the $x$-axis, that is converted into a charge current $\mathbf{J}_{\mathrm{c}}$ via ISHE that is perpendicular to $\mathbf{J}_{\mathbf{s}}$ and to the magnetization vector $\mathbf{M}_{1}$. The spin-flop magnetization process in $\mathrm{FM} / \mathrm{S} / \mathrm{FM}$ trilayer structures could lead to the detection of ISHE voltages at different in-plane orientations to the transversal direction. The external magnetic field $\mathbf{H}$ and the microwave magnetic field $\mathbf{h}_{\mu \omega}$ are applied along the $y$ - and $z$-axes, respectively. 
the FM/S/FM system and the Landau-Lifshitz-Gilbert equation. Considering a trilayer system consisting of two FM layers, whose magnetization and anisotropies are similar, and a metallic spacer $\mathrm{S}$, it is possible to calculate the resonance modes and the magnetization equilibrium angles during the resonance condition. The resonance fields of the SAF and the magnetization equilibrium angles provide a way to determine the relative orientation between the magnetization of each layer as a function of the external magnetic field. Considering two FM layers with the same thickness $d$, the resonance modes of the FM/S/FM system can be obtained from the equations of motion ${ }^{27-29}$

$$
\dot{\mathbf{M}}_{i}=\gamma \mathbf{M}_{i} \times\left(\frac{1}{d} \frac{\partial F_{a}}{\partial \mathbf{M}_{i}}-\frac{\alpha}{\gamma} \frac{\dot{\mathbf{M}}_{i}}{\left|\mathbf{M}_{i}\right|}-\mathbf{h}_{\mu \omega}\right),
$$

where $\mathbf{M}_{i}=\mathbf{M}_{0 i}+\mathbf{m}_{i} e^{i \omega t}$ is the magnetization from the $i$-th layer with $\left|\mathbf{M}_{0 i}\right| \gg\left|\mathbf{m}_{i}\right|, F_{a}$ is the free energy per unit area, $\gamma=g \mu_{B} / \hbar$ is the gyromagnetic ratio and $\alpha$ is the Gilbert damping parameter. We assume that $\left|\mathbf{M}_{i}\right|=M_{i} \approx\left|\mathbf{M}_{0 i}\right|$, due to the smaller value of $\left|\mathbf{h}_{\mu \omega}\right|$ compared to the external magnetic field $\mathbf{H}$. The model also assumes that the magnetization in each layer is in a saturated state during the resonance condition, and also the presence of easy plane anisotropy. The free energy expression of Eq. (1) can be written as

$$
F_{a}=\sum_{i=1}^{2}\left(f_{\text {Zeeman }}^{i}+f_{\text {anis }}^{i}\right)-J_{E} \frac{\mathbf{M}_{1} \cdot \mathbf{M}_{2}}{\left|\mathbf{M}_{1}\right|\left|\mathbf{M}_{2}\right|}
$$

where $f_{\text {Zeeman }}^{i}=-d \mathbf{M}_{i} \cdot \mathbf{H}$ is the Zeeman energy and the term $f_{\text {anis }}^{i}=\frac{d}{2} \mathbf{M}_{i} \mathbf{N}_{i} \mathbf{M}_{i}-d K_{n}^{(i)} \frac{\left(\hat{e}_{\perp} \cdot \mathbf{M}_{i}\right)^{2}}{\left|\mathbf{M}_{i}\right|^{2}}$ corresponds to the anisotropic contribution; $\underline{\mathbf{N}}_{i}, K_{n}^{(i)}$ and $\hat{e}_{\perp}$ being the components of the demagnetizing tensor, the perpendicular anisotropy constant and a unitary vector perpendicular to the plane of the film, respectively. The first term of the anisotropic contribution is related to the effective demagnetizing field associated with $H_{\text {eff }}^{(i)}=4 \pi M_{i}-2 K_{n}^{(i)} / M_{i}$. The last term in Eq. (2) corresponds to the interlayer exchange energy per unit surface, where $J_{E}$ is the coupling constant per unit area and whose sign is negative (positive) for an antiparallel (parallel) coupled system. The exchange energy can also be expressed in magnetic field units according to $H_{E}=\left(\frac{2 J_{E}}{d|\mathbf{M}|}\right)$, for the case $\left|\mathbf{M}_{1}\right|=\left|\mathbf{M}_{2}\right|=|\mathbf{M}|$.

\section{Ferromagnetic coupling}

The oscillation modes for the ferromagnetically coupled situation of two identical FM layers can be obtained in an analytical way only when the external magnetic field is applied parallel or perpendicular to the plane of the trilayer system. When the external magnetic field is applied parallel to the plane of the FM/S/FM layers, the magnetization precession originates two resonance modes: an out-of-phase mode (optical mode) and an in-phase mode (acoustic mode). ${ }^{21,30}$ In the ideal case, where the FM layers are identical, the optical mode intensity should be zero, and only the acoustic mode should be observable. The analytical expressions for the dispersion relation of the acoustic and optic modes when the magnetic field is applied parallel to the trilayer system are shown in the second column of Table I.

The exchange field modifies the dispersion relation revealing that it depends on the magnitude and the sign of the exchange coupling constant. The excitation of the modes, when the external magnetic field is applied parallel to the plane of the films, usually occurs in the saturated state of the system; therefore, the magnetization of both FM layers is parallel during the resonance condition.

\section{Antiferromagnetic coupling}

In contrast to a crystal antiferromagnet, where the exchange interaction occurs between localized spins, antiferromagnetic exchange coupling in a multilayer system couples adjacent layers separated by non-magnetic metallic films. From the equation of motion and the free energy expression, it is also possible to calculate the dispersion relation for both the optical and acoustic modes in the parallel case as a function of the external magnetic field. In this case, the exchange field $H_{E}$ plays an important role in the determination of the functions that describe the relation dispersion $\omega(H)$. For $H<H_{E}$, the analytical expressions of the acoustic and optical modes are shown in the third column of Table I.

In a saturated state, for $H>H_{E}$, the dispersion relation of the acoustic mode takes the same form as in the ferromagnetic exchange coupling case, as shown in Table I. Figure 2(a) shows the simulated relation dispersion of the acoustic mode in a $\mathrm{Co} / \mathrm{S} / \mathrm{Co}$ system for two different values of the exchange field $H_{E}^{1}$ and $H_{E}^{2}$, considering that $H_{E}^{2} \simeq 3 H_{E}^{1}$. The resonance fields of the acoustic modes in the $\mathrm{X}$ band $(9.40 \mathrm{GHz})$ are also indicated in the figure. The dynamic behavior of $\mathrm{Co} / \mathrm{Ru} / \mathrm{Co}$ trilayers studied by FMR evidences that the dispersion relation of the system strongly depends on the exchange coupling constant. ${ }^{21}$

As it was previously mentioned, from Eqs. (1) and (2) that rule the magnetization dynamics of the system, it is also possible to determine the equilibrium angles or the relative orientation between the magnetization of the FM layers that describe the magnetization process of the system. The magnetization process of antiferromagnetic exchange coupled FM layers consists of a spin-flop-like transition, in which the magnetization of each layer is flipped away a certain angle from the external magnetic field, and then monotonically aligned through a continuous rotation of each magnetization until magnetic saturation is reached. The continuous rotation of the magnetization is described by the angle $\phi$, formed by

TABLE I. Analytical expressions of the dispersion relation of the acoustic and optical modes in the ferromagnetic and antiferromagnetic coupling. The magnetic field is applied parallel to the trilayer system.

\begin{tabular}{lcc}
\hline \hline & $\begin{array}{l}\text { FM case and AF } \\
\text { case }\left(H>\left|H_{E}\right|\right)\end{array}$ & $\begin{array}{c}\text { AF } \\
\text { case }\left(H<\left|H_{E}\right|\right)\end{array}$ \\
\hline $\begin{array}{l}\text { Acoustic } \\
\text { mode }\end{array}$ & $\omega^{a c}=\gamma \sqrt{H\left(H+H_{\text {eff }}\right)}$ & $\omega^{a c}=\gamma H \sqrt{1+\frac{H_{\text {eff }}}{\mid H_{E}}}$ \\
$\begin{array}{l}\text { Optical } \\
\text { mode }\end{array}$ & $\omega^{o p}=\gamma \sqrt{\left(H+H_{E}\right)\left(H+H_{E}+H_{\text {eff }}\right)}$ & $\omega^{o p}=\gamma \sqrt{H_{E} H_{\text {eff }}\left[\left(\frac{H}{H_{E}}\right)^{2}-1\right]}$ \\
\hline \hline
\end{tabular}



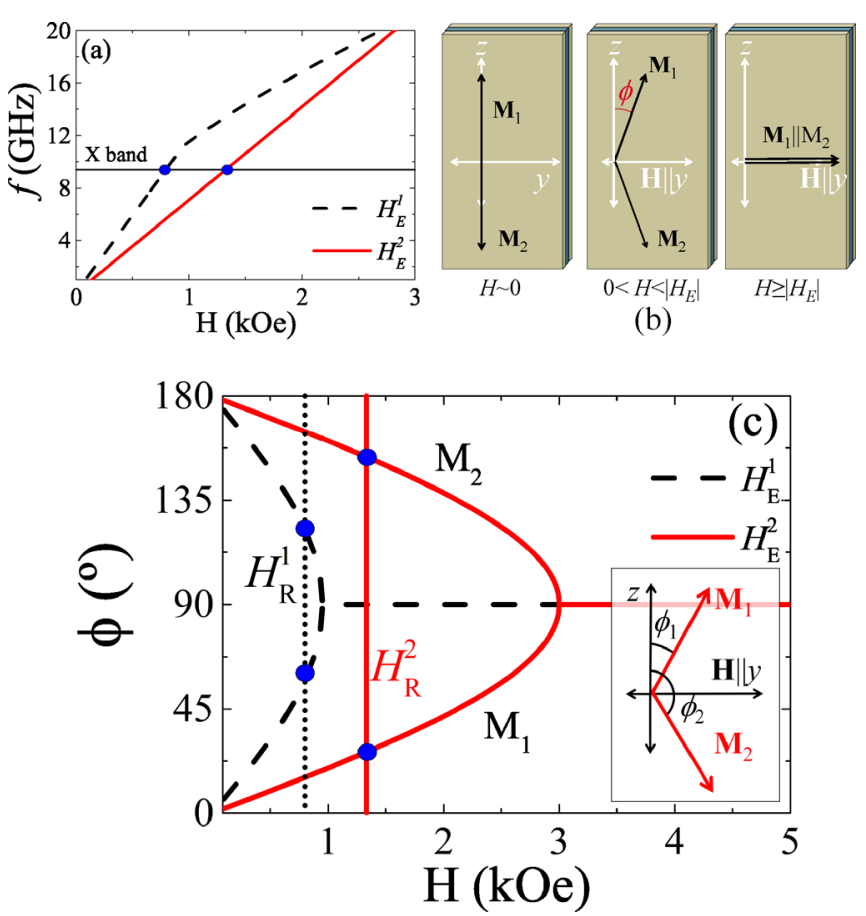

FIG. 2. (a) Simulated dispersion relation $f(H)(\omega=2 \pi f)$ of the acoustic mode for the $\mathrm{Co} / \mathrm{S} / \mathrm{Co}$ system for two different values of the exchange field $\left|H_{E}^{1}\right| \simeq 1000$ Oe (dashed line) and $\left|H_{E}^{2}\right| \simeq 3000$ Oe (solid line). The filled circles indicate the value of the resonance field at $9.40 \mathrm{GHz}$ (X-band). A gyromagnetic factor value $g=2.14$ and an effective shape anisotropy field $H_{\text {eff }}=1.38 \times 10^{4}$ Oe were used. The external magnetic field is applied parallel to the plane of the trilayer system. We assumed that $\left|\mathbf{M}_{1}\right|=\left|\mathbf{M}_{2}\right|$. (b) Schematics of the spin-flop magnetization process in a SAF system. At $H \gtrsim 0$, the magnetization of the layers is antiparallel to each other. When $H$ is oriented perpendicular to the initial magnetization direction, a continuous rotation with increasing field strength occurs towards the field direction. For $H>H_{E}$, the magnetization of both layers is aligned with the external magnetic field. In our analysis of the spin-flop transition, we did not consider an in-plane uniaxial magnetic anisotropy because it is at least one order of magnitude smaller than the interlayer exchange coupling. (c) Simulated magnetization equilibrium angles $\phi$ of a $\mathrm{Co} / \mathrm{S} / \mathrm{Co}$ system as a function of $H$ for the same values of exchange fields $H_{E}^{1}$ (dashed line) and $H_{E}^{2}$ (solid line). Vertical dotted (solid) lines indicate the resonance field $H_{R}^{1}\left(H_{R}^{2}\right)$ of the SAF system with $H_{E}^{1}\left(H_{E}^{2}\right)$. The filled circles indicate the value of the equilibrium angles during the resonance condition in each case. Inset: Schematics of the equilibrium angles $\phi_{1}$ and $\phi_{2}$ of the magnetization vectors during alignment with the external magnetic field.

the magnetization of the FM layers and the $z$ axis, as schematically shown in Fig. 2(b), that depends on $H_{E}\left(\phi=90^{\circ}\right.$ for $H \geq H_{E}$ that corresponds to $\mathbf{M}_{i} \| \mathbf{H}$ ). Figure 2(c) shows a simulation of the magnetization equilibrium angles as a function of the external magnetic field for the same values of exchange fields $H_{E}^{1}$ and $H_{E}^{2}$, where curves above (below) $\phi=90^{\circ}$ correspond to the polar angles of $\mathbf{M}_{2}\left(\mathbf{M}_{1}\right)$. This figure evidences the continuous rotation of the magnetizations $\mathbf{M}_{1}$ and $\mathbf{M}_{2}$ towards the magnetic field direction. Although the FM layers are saturated during the magnetization process, the exchange coupling energy is not overcome until both layers are completely aligned with the external magnetic field at the exchange magnetic field value $\left(H_{E}\right)$. In the antiferromagnetic exchange coupling, the determination of the equilibrium angles is not as simple as in the ferromagnetic case; however, if $\left|\mathbf{M}_{1}\right|=\left|\mathbf{M}_{2}\right|$, the polar angles of the magnetization are supplementaries: $\phi_{1}=\pi-\phi_{2}$ [according to symmetry considerations shown in Fig. 2(b)]. Early experiments in $\mathrm{Co} / \mathrm{Ru} / \mathrm{Co}$ trilayers revealed that Co layers are antiferromagnetically coupled below a certain $\mathrm{Ru}$ thickness $\left(t_{\mathrm{Ru}} \sim 1.2 \mathrm{~nm}\right)$, presenting an oscillatory exchange coupling as a function of the spacer thickness and being possible to achieve large exchange fields, on the order of several $\mathrm{kOe}^{21,31}$

\section{B. Spin pumping and the inverse spin Hall effect in SAF/ML}

In this part, we will discuss the spin pumping process and the inverse spin Hall effect of an antiferromagnetically exchange coupled system into ML with strong SOC. We will consider an antiferromagnetic exchange coupled [FM/S/ $\mathrm{FM}] / \mathrm{ML}$ system as our [SAF]/ML heterostructure for the analysis of the SP-ISHE process. For the spin pumping description in the SAF/ML, we will also take into consideration the case when the resonance field value $H_{R}$ of the trilayer system is below the exchange field or $H_{R}<H_{E}$ for some fixed frequency, i.e. the resonance condition occurs when the FM layers are not completely aligned with the external magnetic field. To contrast the SP-ISHE in SAF-ML with the conventional case, we will briefly discuss the spin pumping of a FM/ML bilayer system and the detection of the generated spin current via ISHE.

\section{Single Co layer injection}

The most common system used for spin current generation and detection via SP-ISHE is a FM/ML bilayer. In resonance condition, a pure spin current is injected into the metallic layer due to the interfacial relaxation process mediated by the conduction electrons of the ML. The instantaneous spin pumping current $\mathbf{J}_{\mathbf{s}}$, with a unit vector of the spin current polarization $\mathbf{s}$, is given by $J_{\mathbf{s}} \hat{\mathbf{s}}=\frac{\hbar}{8 \pi} \operatorname{Re}\left(2 g^{\uparrow \downarrow}\right)\left[\mathbf{m} \times \frac{\partial \mathbf{m}}{\partial t}\right]$, where $\mathbf{m}$ is the unit magnetization vector and $\operatorname{Re}\left(g^{\uparrow \downarrow}\right)$ is the real part of the spin mixing conductance. The pure spin current is converted into a transversal charge current via the inverse spin Hall effect according to $\mathbf{J}_{\mathbf{c}}=\Theta_{S H}\left(\frac{-2 e}{\hbar}\right) \mathbf{J}_{\mathbf{s}} \times \boldsymbol{\sigma}$. A schematic of the relative orientation between the electrical field and the magnetization vector associated with the SP-ISHE mechanism in a FM/ML system is shown in Fig. 3(a). The time averaged $\mathbf{J}_{\mathbf{s}}$ has a non-zero component only in the direction of the applied magnetic field, and because the electrical field generated by the ISHE is transversal to the magnetization vector of the FM film, an in-plane angular variation of the ISHE voltages fits to a cosine function as shown in Fig. 3(b). This dependence was demonstrated by previous authors ${ }^{32,33}$ by measuring the ISHE signals varying the relative orientation between the external magnetic field and the electrical contact direction for the detection of the ISHE around an axis perpendicular to the plane of the sample [angle $\alpha$ in Fig. 3(a)].

Previous works on bilayers such as $\mathrm{Fe}_{20} \mathrm{Ni}_{80} / \mathrm{Ta}$, ${ }^{15}$ $\mathrm{Fe}_{20} \mathrm{Ni}_{80} / \mathrm{Pt},{ }^{32,33} \mathrm{YIG} / \mathrm{Ta}^{16}$ and $\mathrm{YIG} / \mathrm{Pt}^{34}$ not only set the foundation for a better understanding of the detection of pure spin currents, but also evidenced the influence of particular electronic transport effects such as anomalous Hall effects, anisotropic magnetoresistance and spin Hall magnetoresistance on 

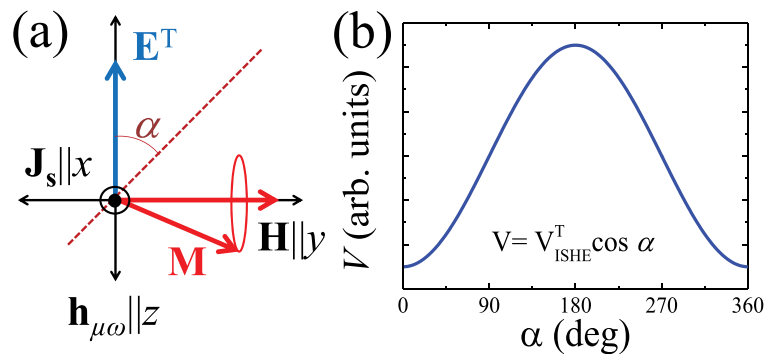

FIG. 3. (a) Schematic of the ISHE electrical field $\mathbf{E}^{\mathrm{T}}$ and the magnetization vector $\mathbf{M}$ during the spin pumping process in a FM/ML system. In the resonance condition, magnetization of the FM layer (parallel to the external magnetic field and precession around the $y$-axis) pumps a spin current $\mathbf{J}_{\mathbf{s}}$ into the metallic layer parallel to the $x$-axis (not shown), that is converted into a transversal charge current $\mathbf{J}_{\mathbf{c}}$ via ISHE. The dashed line indicates an arbitrary axis as a reference for the in-plane ISHE measurements through the angle $\alpha$. (b) In-plane angular dependence of the ISHE signal for the FM/NM bilayer system.

the spin Hall voltages ${ }^{16,32,33}$ that affect the in-plane angular dependence of the ISHE signal. For instance, in some cases, the detected dc voltages in SP-ISHE experiments show a more pronounced asymmetric behavior due to the anisotropic magnetoresistance in the FM films ${ }^{33}$ that causes difficulty in the interpretation of in-plane measurements.

\section{Antiferromagnetically coupled Co layers: Adjacent layer injection}

The SP-ISHE process in SAF/ML heterostructures strongly depends on the interlayer exchange coupling between the FM layers. As it was previously mentioned, the exchange field $H_{E}$ influences the magnetization dynamics and the resonance frequency dependence on the external magnetic field. On the one hand, if the FM layers of the SAF are ferromagnetically coupled, the magnetization vectors are parallel to each other along the external magnetic field during resonance conditions and the spin pumping process is similar to a FM/ML system. A pure spin current will be injected into the metallic layer and converted into a transversal charge current [parallel to the $z$ axis according to Fig. 3(a)]. On the other hand, if the FM layers are antiferromagnetically exchange coupled, the resonance condition could occur before the magnetization of the FM layers is completely aligned with the external magnetic field, as schematically shown in Figs. 2(c) and 4(a). Because the spin pumping is an interfacial process, we will consider that the pure spin current is generated only from the FM layer adjacent to the ML, and the polarization of the spin current will be determined by the corresponding magnetization vector $\mathbf{M}_{1}$. The magnetization $\mathbf{M}_{2}$ of the FM which is not adjacent to the ML will not be considered in the spin pumping process. The orientation of the magnetization $\mathbf{M}_{1}$ during the spin pumping is determined by the equilibrium angle $\phi$ obtained from Eqs. (1) and (2). Therefore, the polarization vector $\mathbf{s}$ of the spin current also depends on $\phi$ because the precession of $\mathbf{M}_{1}$ at $H=H_{R}<H_{E}$ is misaligned with the external magnetic field.

The spin current injected into the ML with the magnetization vector tilted at an angle $\phi$ will be converted into a charge current via ISHE with the transversal electric field

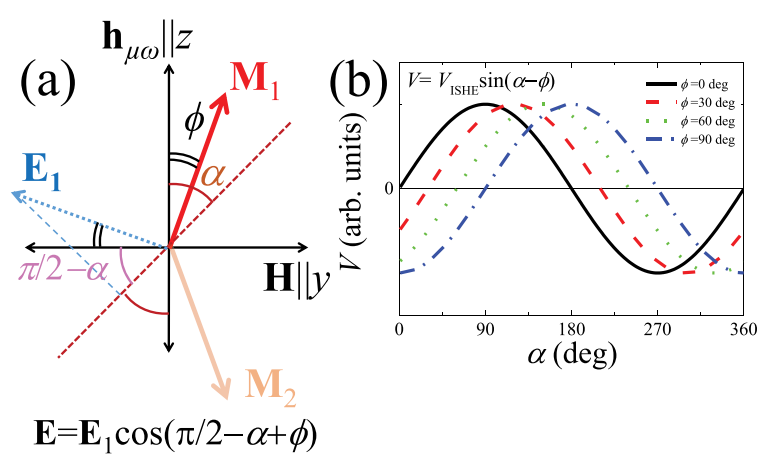

FIG. 4. (a) Schematics of the ISHE electrical field $\mathbf{E}_{1}$ and the magnetization vector $\mathbf{M}_{1}$ during the spin pumping process in a SAF/ML system. In the resonance condition, the magnetization of the FM layer pumps a spin current $\mathbf{J}_{\mathbf{s}}$ into the metallic layer that is converted into a transversal charge current $\mathbf{J}_{\mathbf{c}}$ via ISHE. (b) In-plane angular dependence of the ISHE signal for the FM/ NM bilayer system. In this picture, the magnetization $\mathbf{M}_{2}$ from the layer which is not adjacent to the ML does not contribute to the spin pumping process.

vector $\mathbf{E}_{1}$ also tilted at an angle $\phi$ from the coordinate axis according to Fig. 4(a). Conventional measurements of the ISHE signals place the electrical contacts in a transversal orientation from the external magnetic field (along the $z$ axis in this case). Performing electrical measurements in that configuration will only allow us to detect the projection of the ISHE electric field along the $z$ axis. Our analysis of the SPISHE process in SAF/ML indicates that the maximum value of the ISHE signal can be tuned by performing in-plane measurements of the induced voltages as a function of an arbitrary angle $\alpha$ on the plane of the films. The main feature of the in-plane angular dependence of the ISHE signal is that it can be fitted to a sine function shifted by the value of $\phi$. According to Fig. 4(a), if we place the electrical contacts along the $\mathbf{M}_{1}$ direction, the ISHE signal will be zero due to the induced electrical field vector being perpendicular to the magnetization, and therefore to the line of the electrical contacts. However, following the same reasoning, the maximum value of the ISHE signal will be found at $\pi / 2+\phi$ (or $\phi-\pi / 2$ ), as shown in Fig. 4(b).

In this particular case, it can also be deduced from Fig. 4(a) that comparing the magnitude of the transversal and longitudinal components of the ISHE signals, corresponding to the cases where $\alpha=0$ and $\alpha=90^{\circ}$ according to the schematic of the ISHE electrical field shown in Fig. 4(a), the value of the equilibrium angle of the magnetization during the resonance condition can be estimated. The transversal and longitudinal components of the ISHE voltage labeled as $V_{\text {ISHE }}^{\mathrm{T}}$ and $V_{\text {ISHE }}^{\mathrm{L}}$, respectively, are the result of measuring the ISHE electrical field projection along the $z$ - and $y$-axes and are related to the equilibrium angles of the magnetization through the following expression:

$$
V_{\mathrm{ISHE}}^{\mathrm{T}} / V_{\mathrm{ISHE}}^{\mathrm{L}}=\tan \phi .
$$

The schematic of Fig. 4(a) and the $\phi$-dependence with the external magnetic field allow us to predict the behavior of $V_{\text {ISHE }}^{\mathrm{T}}$ and $V_{\text {ISHE }}^{\mathrm{L}}$ for different exchange coupling strengths. Although it was not considered in Fig. 4(b), it is important to mention that the magnetization equilibrium angle will also 
affect the magnitude of the spin current $\mathbf{J}_{\mathbf{s}}$ injected into the ML because the product $\left[\mathbf{m} \times \frac{\partial \mathbf{m}}{\partial t}\right]$ is also influenced by the magnetization equilibrium angle $\phi$.

It is important to notice that in the calculation of the resonance modes and the equilibrium angles in the FM/S/FM, we are not considering the influence of the spin current pumped from one FM layer on the magnetization dynamics of the other FM layer. Although the spin diffusion lengths are slightly larger than the required thickness to achieve AFM coupling, the spin transport in ferromagnets is a more complex problem because the scattering process and length scales are spin dependent. If we assume that part of the spin current injected from the FM layer which is not adjacent to the ML propagates through the FM/ML interface with an attenuated amplitude, it is possible with our model to estimate the inplane angular dependence of the ISHE signal for the [SAF]/ ML system. Considering that an additional ISHE electric field $\mathbf{E}_{2}$ is generated by the magnetization $\mathbf{M}_{2}$ [according to the schematics of Fig. 4(a)] of the layer which is not in contact with the ML, the total electric field will be of the form $\mathbf{E}=\mathbf{E}_{1} \cos (\pi / 2-\alpha+\phi)+\mathbf{E}_{2} \cos (\pi / 2-\alpha-\phi)$, with $\left|\mathbf{E}_{2}\right|$ $<\left|\mathbf{E}_{1}\right|$, and the amplitude of the ISHE voltages will be slightly modified depending on the factor $\left|\mathbf{E}_{2}\right| /\left|\mathbf{E}_{1}\right|$.

\section{Tuning the sign of the ISHE in SAF/ML}

For the analysis of the magnetization dynamics, we have considered that both FM layers in the SAF system have identical magnetization and anisotropy values. From the results, we concluded that the spin-flop magnetization process can lead to the generation of pure spin currents polarized in arbitrary directions as a consequence of the misalignment of the magnetization of the FM during the resonance condition. In general, the fabrication of two identical layers is quite difficult using standard deposition methods, and small variations in the magnetic moments or the anisotropies of the layers are unavoidable. This can be used to select the sign of the ISHE voltage following an appropriate field protocol.

To picturize this situation, we can consider that the FM layers are not perfectly identical, and one of the layers has an in-plane anisotropy larger than the other, with their corresponding easy anisotropy axes parallel to each other. It is important to mention that in this picture, the magnetic moments of both layers are equal. In this case, after the application of a large external magnetic field along the $+y$ or $-y$ direction, the magnetization of the FM layer with a larger anisotropy constant would rotate following the direction of its corresponding easy axis. Figure 5(a) shows a schematic of the magnetization process considering that the magnetization $\mathbf{M}_{1}$ of the layer which is in contact with the ML has a larger in-plane anisotropy (represented by $\mathrm{EA}_{1}$ ) than the other layer. After the application of a magnetic field along the $-y$ direction being large enough to overcome the exchange field, the alignment of the magnetization vector $\mathbf{M}_{1}$ is determined by the easy axis $\mathrm{EA}_{1}$ and will rotate in a counter-clockwise direction along the lower quadrant according to Fig. 5(b). On the other hand, if the SAF is saturated along the $+y$ direction [Fig. 5(c)], the rotation of $\mathbf{M}_{1}$ will also follow a counter-clockwise rotation but towards the

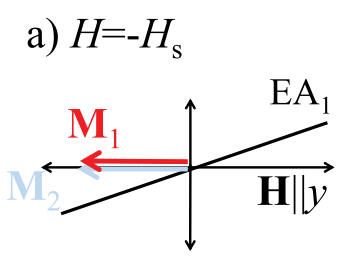

b) $H=H_{\mathrm{R}}$

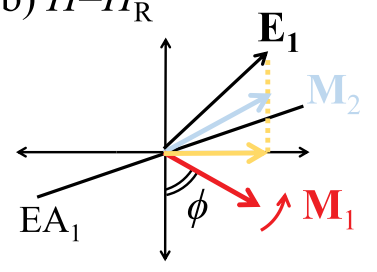

c) $H=+H_{\mathrm{s}}$

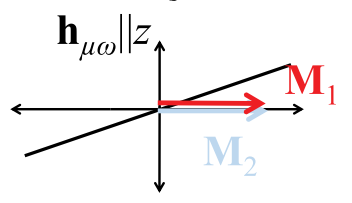

d) $H=H_{\mathrm{R}}$

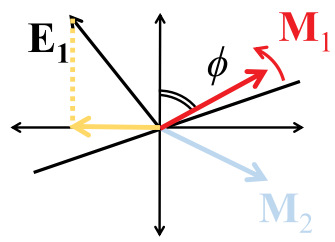

FIG. 5. Schematic of the mechanism for the tuning of the sign of the ISHE voltage in the SAF/ML system. Considering that layer 1 is more anisotropic than layer 2, it is possible to change the direction of magnetization $\mathbf{M}_{1}$ during the resonance condition. (a) Magnetization of the layers $\mathbf{M}_{1}$ and $\mathbf{M}_{2}$ after the application of a large external magnetic field $\left(H=H_{s}\right)$ along the $-y$ direction. (b) As we increase $H, \mathbf{M}_{1}$ will rotate towards the direction of the easy axis $\mathrm{EA}_{1}$ (solid line). During the SP-ISHE measurement, the projection of the ISHE electric field vector along the $z$ axis or $V_{\text {ISHE }}^{\mathrm{L}}$ is positive. (c) Magnetization of the layers $\mathbf{M}_{1}$ and $\mathbf{M}_{2}$ after the application of a large external magnetic field along the $+y$ direction. (d) As we decrease $H, \mathbf{M}_{1}$ would rotate towards the direction of $\mathrm{EA}_{1}$, however, in this case, the projection of the ISHE electric field along the $z$-axis has a different sign, therefore, $V_{\text {ISHE }}^{\mathrm{L}}$ is negative.

direction of $\mathrm{EA}_{1}$ along the upper quadrant as shown in Fig. 5(d). In both cases, $\mathbf{M}_{2}$ will rotate simultaneously with $\mathbf{M}_{1}$ but in an opposite direction due to the IEC coupling.

In resonance condition (at $H=H_{R}$ ), the magnetization $\mathbf{M}_{1}$ will pump a pure spin current that generates an electric field $\mathbf{E}_{1}$ due to the ISHE. In both cases, $\mathbf{E}_{1}$ will point in a transversal direction to $\mathbf{M}_{1}$ as shown in Figs. 5(b) and 5(d) and will have the same magnitude, but different directions. This could be evidenced experimentally by measuring the longitudinal and transversal components of the ISHE signals. In the cases described from Figs. 5(a) to 5(d), we deduced that the transversal components $V_{\text {ISHE }}^{\mathrm{T}}$ of the ISHE signals will remain unchanged but we expected a change of sign of the longitudinal component $V_{\text {ISHE }}^{\mathrm{L}}$.

The change of sign of the ISHE voltage can also be explained by considering that the in-plane anisotropy easy axes are parallel and have the same magnitude for both ferromagnetic layers, but the total magnetic moment of the layers is slightly different (this situation is commonly obtained during the growth of thin films with undesired different thickness). In this picture, a variation of the total magnetic moment between the layers also breaks the symmetry of the magnetization rotation during SP-ISHE measurements of SAF/ML systems. In this case, the Zeeman energy will determine the magnetic moment rotation of the magnetic moment in each layer. The layer with a smaller magnetic moment will always rotate towards the direction of the in-plane anisotropy easy axis because the Zeeman energy of the other layer will tend to align the magnetic moment with the external magnetic field.

This result can be interpreted as an additional mechanism to control the polarization vector $\mathbf{s}$ of the pure spin current $\mathbf{J}_{\mathbf{s}}$ pumped into the ML and consequently, the sign of 
the ISHE signal by properly preparing the initial magnetization configuration.

\section{CONCLUSIONS}

In conclusion, we have proposed a phenomenological model to describe the spin currents generated by artificial antiferromagnetically exchange coupled systems and heavy metal layers with strong spin-orbit coupling. Exploiting the magnitude of the exchange coupling and the spin-flop transition in the magnetization process, it is possible to produce spin currents polarized in arbitrary directions. The spin-flop transition of the SAF/NM system originates pure spin currents whose polarization vector depends on the exchange field and the magnetization equilibrium angles. Considering that the layers have different in-plane anisotropy values, we discuss an additional way to control the polarization of the pure spin current injected into the ML by changing the initial conditions of the external magnetic field in the SP-ISHE experiments. The variation of anisotropies in the FM layers leads to a change in the magnetization vector rotation that permits the control of the sign of the ISHE voltage components.

To our knowledge, a study that discusses the behavior of a SAF system as a spin current generator via spin pumping has not yet been reported. Spin current injection in the $\mathrm{GHz}$ region produced by the SAF/ML systems could contribute to the development of new high frequency spintronic devices. Our results can contribute to important advances in hybrid spintronic devices with new functionalities, particularly the ability to control microscopic parameters such as the polarization direction of the pure spin current through the variation of macroscopic parameters, such as the thickness of the NM in antiferromagnetic exchange coupled [FM/S/FM]/ML systems, or the external magnetic field.

\section{ACKNOWLEDGMENTS}

The authors would like to thank R. Benavides, C. Pérez, and M. Guillén for their extraordinary technical support. This work was partially supported by CONICET (Grant No. PIP 201501-00213), ANPCyT (Grant No. PICT 2013-0401), and U. N. Cuyo (Grant No. 06/C484), all from Argentina.

${ }^{1}$ T. Jungwirth, X. Marti, P. Wadley, and J. Wunderlich, Nat. Nanotechnol. 11, 231 (2016)

${ }^{2}$ J. Sklenar, W. Zhang, M. B. Jungfleisch, W. Jiang, H. Saglam, J. E. Pearson, J. B. Ketterson, and A. Hoffmann, AIP Adv. 6, 055603 (2016).

${ }^{3}$ R. Cheng, J. Xiao, Q. Niu, and A. Brataas, Phys. Rev. Lett. 113, 057601 (2014).

${ }^{4} \varnothing$. Johansen and A. Brataas, Phys. Rev. B 95, 220408 (2017).

${ }^{5}$ A. Brataas, H. Skarsvåg, E. G. Tveten, and E. Løhaugen Fjærbu, Phys. Rev. B 92, 180414 (2015).
${ }^{6}$ H. Wang, C. Du, P. C. Hammel, and F. Yang, Phys. Rev. Lett. 113, 097202 (2014).

${ }^{7}$ V. Tshitoyan, C. Ciccarelli, A. P. Mihai, M. Ali, A. C. Irvine, T. A. Moore, T. Jungwirth, and A. J. Ferguson, Phys. Rev. B 92, 214406 (2015). ${ }^{8}$ H. Saglam, W. Zhang, M. B. Jungfleisch, J. Sklenar, J. E. Pearson, J. B. Ketterson, and A. Hoffmann, Phys. Rev. B 94, 140412 (2016).

${ }^{9}$ S. M. Wu, W. Zhang, A. Kc, P. Borisov, J. E. Pearson, J. S. Jiang, D. Lederman, A. Hoffmann, and A. Bhattacharya, Phys. Rev. Lett. 116, 097204 (2016).

${ }^{10}$ S. Seki, T. Ideue, M. Kubota, Y. Kozuka, R. Takagi, M. Nakamura, Y. Kaneko, M. Kawasaki, and Y. Tokura, Phys. Rev. Lett. 115, 266601 (2015).

${ }^{11}$ J. B. S. Mendes, R. O. Cunha, O. Alves Santos, P. R. T. Ribeiro, F. L. A. Machado, R. L. Rodríguez-Suárez, A. Azevedo, and S. M. Rezende, Phys. Rev. B 89, 140406 (2014)

${ }^{12}$ R. Khymyn, I. Lisenkov, V. S. Tiberkevich, A. N. Slavin, and B. A. Ivanov, Phys. Rev. B 93, 224421 (2016).

${ }^{13}$ K. Ando, S. Takahashi, J. Ieda, Y. Kajiwara, H. Nakayama, T. Yoshino, K. Harii, Y. Fujikawa, M. Matsuo, S. Maekawa, and E. Saitoh, J. Appl. Phys. 109, 103913 (2011).

${ }^{14}$ J.-C. Rojas-Sánchez, N. Reyren, P. Laczkowski, W. Savero, J.-P. Attané, C. Deranlot, M. Jamet, J.-M. George, L. Vila, and H. Jaffrès, Phys. Rev. Lett. 112, 106602 (2014).

${ }^{15}$ J. E. Gómez, B. Zerai Tedlla, N. R. Álvarez, G. Alejandro, E. Goovaerts, and A. Butera, Phys. Rev. B 90, 184401 (2014).

${ }^{16}$ C. Hahn, G. D. Loubens, O. Klein, M. Viret, and J. B. Youssef, Phys. Rev. B 87, 174417 (2013).

${ }^{17}$ G. Y. Luo, J. G. Lin, W.-C. Chiang, and C.-R. Chang, Sci. Rep. 7, 6612 (2017).

${ }^{18}$ M. Wahler, N. Homonnay, T. Richter, A. Mller, C. Eisenschmidt, B. Fuhrmann, and G. Schmidt, Sci. Rep. 6, 28727 (2016).

${ }^{19}$ J. E. Gómez, J. M. Vargas, L. Avilés-Félix, and A. Butera, Appl. Phys. Lett. 108, 242413 (2016).

${ }^{20}$ S. S. P. Parkin, N. More, and K. P. Roche, Phys. Rev. Lett. 64, 2304 (1990).

${ }^{21}$ Z. Zhang, L. Zhou, P. E. Wigen, and K. Ounadjela, Phys. Rev. B 50, 6094 (1994).

${ }^{22}$ W. Alayo, E. Baggio-Saitovitch, F. Pelegrini, and V. P. Nascimento, Phys. Rev. B 78, 134417 (2008).

${ }^{23}$ J. Unguris, R. J. Celotta, and D. T. Pierce, Phys. Rev. Lett. 79, 2734 (1997).

${ }^{24}$ D. E. González-Chávez, R. Dutra, W. O. Rosa, T. L. Marcondes, A. Mello, and R. L. Sommer, Phys. Rev. B 88, 104431 (2013).

${ }^{25}$ L. Avilés-Félix, A. Butera, and J. E. Gómez, Appl. Phys. Lett. 110, 052402 (2017).

${ }^{26}$ J. E. Gómez, M. Guillén, A. Butera, and N. P. Albaugh, Rev. Sci. Instrum. 87, 024705 (2016).

${ }^{27}$ J. Smit and H. Beljers, Philips Res. Rep. 10, 113 (1955).

${ }^{28}$ J. Gómez, J. L. Weston, and A. Butera, J. Appl. Phys. 100, 053908 (2006).

${ }^{29}$ J. Gómez, J. L. Weston, and A. Butera, Phys. Rev. B 76, 184416 (2007).

${ }^{30}$ P. E. Wigen, Z. Zhang, L. Zhou, M. Ye, and J. A. Cowen, J. Appl. Phys. 73, 6338 (1993).

${ }^{31}$ Z. Zhang, L. Zhou, P. E. Wigen, and K. Ounadjela, J. Appl. Phys. 75, 6434 (1994).

${ }^{32}$ O. Mosendz, V. Vlaminck, J. E. Pearson, F. Y. Fradin, G. E. W. Bauer, S. D. Bader, and A. Hoffmann, Phys. Rev. B 82, 214403 (2010).

${ }^{33}$ A. Azevedo, L. H. Vilela-Leão, R. L. Rodríguez-Suárez, A. F. Lacerda Santos, and S. M. Rezende, Phys. Rev. B 83, 144402 (2011).

${ }^{34}$ H. Nakayama, M. Althammer, Y. T. Chen, K. Uchida, Y. Kajiwara, D. Kikuchi, T. Ohtani, S. Geprägs, M. Opel, S. Takahashi, R. Gross, G. E. W. Bauer, S. T. B. Goennenwein, and E. Saitoh, Phys. Rev. Lett. 110, 206601 (2013). 